\title{
Analysis of paint thickness based on flash pulse thermography
}

\author{
by L. Muzika* and M. Švantner* \\ * University of West Bohemia, New Technologies - Research Center, Univerzitní 8, Plzeň, Czech Republic, \\ muzika@ntc.zcu.cz, msvantne@ntc.zcu.cz
}

\begin{abstract}
Coating thickness (thermal barrier coatings, anticorrosion paints, etc.) can influence its properties and therefore the thickness is often measured in industrial application. This contribution describes fast areal thickness estimation based on flash pulse thermography. Specimens sprayed by a black paint on AISI 304 substrates were used for a demonstration of the thickness estimation. Two different types of post-processing and thickness estimation were used: functional calibration with FFT (phase) and analytical model. Both procedures were compared and both provided satisfactory results with maximum error of thickness estimation less than $10 \%$. Advantages and disadvantages of proposed techniques are discussed.
\end{abstract}

\section{Introduction}

The thickness is one of the most important parameters when dealing with paints. Paint used in industry usually have except a decorative purpose also functional purpose, e.g. a protection against corrosion, acids, etc. The problem is when inappropriate amount of a paint is applied which causes that required properties (e.g. anticorrosion) are not met.

Nondestructive thickness measurement can be performed by many different techniques. One of the most used are [1]: measurement with ultrasound testing, measuring with devices based on magnetic induction or eddy current. All of those techniques are limited to some materials and they took readings from one point. When readings of an area need to be acquired, the measurement process can be time demanding. Because of that a technique which would provide readings from the whole surface could speed up the thickness measurement. This contribution describes two techniques for a planar thickness determination based on flash pulse thermography.

\section{Thickness determination with flash pulse thermography}

Flash pulse thermography (FPT) is an infrared non-destructive testing inspection method. The technique is based on an excitation of tested subject by a flash lamp with one short pulse (few $\mathrm{ms}$ ). The temperature response to this pulse is recorded by an infrared camera. This response can show changes in heat transfer caused by defects (for example cracks, delamination but also inhomogeneous thickness of a paint).

Two approaches can be used to estimate a paint thickness. First approach is based on functional dependency between thickness and some obtained parameter (e.g. phase when FFT [2] is applied to a thermographic sequence). With this approach an equation between depth and the parameter must be found. This can be done via calibration samples, nonetheless the equation is valid just for a material of paint and substrate. Second approach is based on comparison of temperature response obtained via IR measurement with a solution of an analytical or a numerical model. In that case thermal properties of both paint and substrate must be known, as well as the thickness of the substrate and energy. The problem is that many of those initial data are unknown. One of the goal of this contribution was to verify if both of those techniques can be used for determination of paint thickness.

\section{Experimental setup}

Two specimens from AISI 304 steel with dimensions $100 \times 50 \times 2 \mathrm{~mm}$ were painted with a black paint (LabIR HERP-LT-MWIR-BK-11). The paint was sprayed by hand on the surface of specimens. Experiments were performed for both samples. IR camera FLIR SC 7650 was used for thermographic measurement. The framerate was $250 \mathrm{~Hz}$ and $4 \mathrm{~s}$ were recorded. Specimens were excited by $6000 \mathrm{~J}$ flash lamp. Centre of the tested specimen was in the centre of the flash lamp during measurements. The flash lamp was $38 \mathrm{~cm}$ from specimens and was perpendicular to the specimens.

FFT was performed on de-noised data (fitting with function). Then a fast Fourier transform was applied and a phase was calculated. Suitable frame was used for calibration between thickness and phase. The dependency was described by the equation.

$$
z_{c}=C_{1} \cdot \Phi^{3}+C_{2} \cdot \Phi^{2}+C_{3} \cdot \Phi+C_{4}
$$

, where $z_{c}$ is thickness, $C_{I^{-}} C_{4}$ are constants and $\Phi$ is the phase angle. 
The analytical model was based on thermal quadrupoles [6]. It was one of the simplistic model which can be used. Known parameter, which were needed for calculations were substrate's: thickness $(2 \mathrm{~mm})$, thermal diffusivity $\left(4 \mathrm{E}-6 \mathrm{~m}^{2} / \mathrm{s}\right)$, thermal conductivity $\left(16.2 \mathrm{~W} \cdot \mathrm{m}^{-1} \cdot \mathrm{K}^{-1}\right)$ and paint's: thermal diffusivity $\left(2.22 \mathrm{E}-7 \mathrm{~m}^{2} / \mathrm{s}\right)$ and thermal conductivity $\left(0.75 \mathrm{~W} \cdot \mathrm{m}^{-1} \cdot \mathrm{K}^{-1}\right)$. Unknown parameters were thickness of paint and energy. The energy was obtained via measurement of sample 1 (thickness of coating was known based on a point thickness meter measurement). Temperature responses from specimen 2 were compared with analytical solutions with different thickness of paint. The thickness of the analytical solution which had the best match with the measurement was determined as the thickness of the paint at given place.

\section{Results}

The calibration equation was obtained from a comparison of flash pulse measurement of specimen 1 and measurement done by the thickness meter. The coefficients C1-C4 were 464, 219.2, 58.04, 45.11. Figure 1 shows thickness maps obtained via calibration with FFT and thickness map obtained via analytical model.

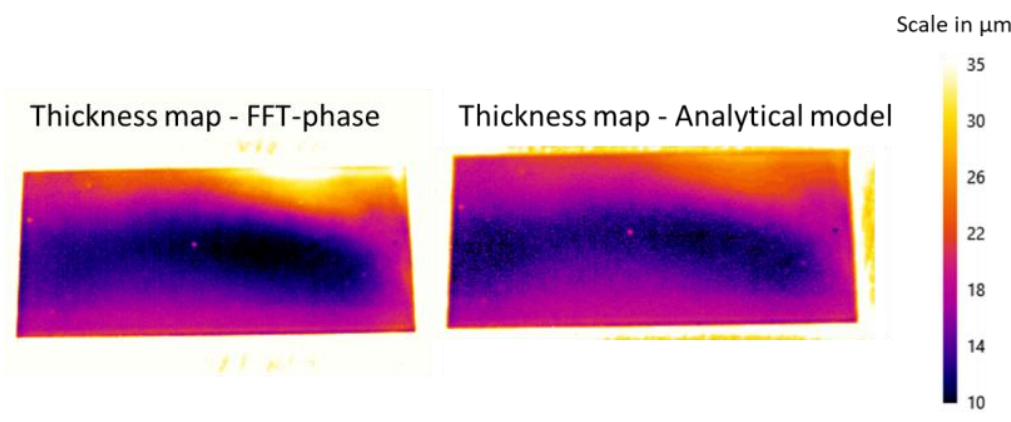

Fig. 1 Thickness maps obtained via FFT and via analytical model

Both results showed approximately same thickness map. Most importantly it was possible to clearly indicate parts of the sample with different paint thickness. Maximum relative error of thickness estimation for both techniques was $8 \%$ compared to measurement by thickness meter Positector 6000.

\section{Conclusions}

Two types of thickness estimation techniques based on flash pulse thermography were described. Both techniques showed satisfactory paint thickness estimation. Paint thickness of the specimen was from 10 to $30 \mu \mathrm{m}$ and maximum relative error was $8 \%$ (which corresponded to $2 \mu \mathrm{m}$ difference compared to standard measurement with Positector 6000).

In general, the biggest advantage of using flash pulse thermography for paint or other coating thickness estimation is that thickness map of whole surface can be made. Most of standard non-destructive thickness estimation methods take reading from one point only. Another advantage is that one measurement with flash pulse thermography covered $5 \times 10 \mathrm{~cm}$ sample and took $4 \mathrm{~s}$, this correspond to time of 2-4 measurement with point thickness meter. That means the measurement can be speed up with pulse thermography.

Calibration approach seems to be more practical than analytical model for real application due to easier implementation of the whole procedure. The problem with calibration is that it is valid just for one material of paint and substrate and as well the thickness of substrate. For calibration approach the FFT-phase was used. Nonetheless other techniques can be used as well and may produce satisfactory results. Searching for most suitable pre-processing for thickness estimation should be the next part of the research as well as definition of materials which can be tested with flash pulse thermography. estimation

The results show that flash pulse thermography could be a viable high-speed tool for an areal paint thickness

\section{REFERENCES}

[1] Mezghani S, Perrin E, Bodnar J L, Marthe J, Cauwe B, Vrabie V and Samples A 2015 Evaluation of Heterogeneity of Paint Coating on Metal Substrate Using Laser Infrared Thermography and Eddy Current [online]. 9(5), 837-842. ISSN 1359-6640. doi:10.1039/B919800H

[2] Shrestha R and Kim W 2018 Evaluation of coating thickness by thermal wave imaging: A comparative study of pulsed and lock-in infrared thermography - Part II: Experimental investigation. Infrared Physics and Technology [online]. 92(May), 24-29. ISSN 13504495. doi:10.1016/j.infrared.2018.05.001

[3] D. Maillet, S. André, J.-C. Batsale, A. Degiovanni, C. Moyne, Thermal Quadrupoles: Solving the Heat Equation through Integral Transforms. John Wiley and Sons, New-York, 2000. 